\title{
Patient factors and their impact on neutropenic events: a systematic review and meta-analysis
}

\author{
Pinkie Chambers ${ }^{1}$ (D) Yogini Jani $^{1} \cdot$ Li Wei $^{2} \cdot$ Emma Kipps $^{3} \cdot$ Martin D. Forster $^{4} \cdot$ lan C. K. Wong ${ }^{2,5}$
}

Received: 13 December 2018 / Accepted: 22 March 2019 / Published online: 16 April 2019

(C) The Author(s) 2019

\begin{abstract}
Background Neutropenia is associated with an increased risk of mortality and hospitalisation. Strategies, including the prescribing of colony-stimulating growth factors (CSFs), are adopted when a high risk (>20\%) of neutropenic complications are seen in the clinical trial setting. With a diverse treatment population that may differ from the patient groups recruited to studies, appropriate prescribing decisions by clinicians are essential. At present, results are conflicting from studies evaluating the risks of certain patient attributes on neutropenic events; we aimed to aggregate these associations to guide future management.

Design A systematic review with a meta-analysis was conducted using the Preferred Reporting Items for Systematic Reviews and Meta-Analysis (PRISMA) statement. Studies were identified through a literature search using MEDLINE, EMBASE and Cumulative Index to Nursing and Allied Health Literature (CINAHL) databases from inception to December 1, 2017. Studies were included into a meta-analysis if they adjusted for confounders; analyses were conducted in STATA v 15.1 SE.

Results A total of 4415 articles were retrieved by the search with 37 meeting the inclusion criteria and 12 eligible for metaanalysis. Meta-analysis was conducted for increasing age and yielded a pooled odds ratio of $1.39\left(1.11,1.76, \mathrm{I}^{2}=24.1 \%\right)$, in our subgroup analysis of 4814 patients. Odds ratios for studies were pooled that reported associations for one co-morbidity compared to none and resulted in an overall odds of 1.54 (CI 1.09-2.09, $\mathrm{I}^{2}=13.1 \%$ ), including 9189 patients in total.

Conclusions Results can enhance current guidance in prescribing primary prophylaxis for treatments that either fall marginally under the internationally recognised $20 \%$ neutropenia risk.
\end{abstract}

Keywords Neutropenia $\cdot$ Cancer $\cdot$ Chemotherapy $\cdot$ Neutropenic sepsis $\cdot$ Risk

Electronic supplementary material The online version of this article (https://doi.org/10.1007/s00520-019-04773-6) contains supplementary material, which is available to authorized users.

Pinkie Chambers

p.chambers@ucl.ac.uk

Yogini Jani

y.jani@ucl.ac.uk

Li Wei

1.wei@ucl.ac.uk

Emma Kipps

e.kipps@nhs.net

Martin D. Forster

martin.forster1@nhs.net

Ian C. K. Wong

i.wong@ucl.ac.uk
1 UCLH-UCL Centre for Medicines Optimisation Research and Education, Pharmacy Department, University College London Hospitals NHS Foundation Trust, 235 Euston Road, London NW1 2BU, UK

2 UCLH-UCL Centre for Medicines Optimisation Research and Education, UCL School of Pharmacy, 29-39, Brunswick Square, London WC1N 1AX, UK

3 The Royal Marsden Hospital, Fulham Road, London SW3 6JJ, UK

4 UCL Cancer Institute, Department of Oncology, University College London Hospitals NHS Foundation Trust, 72 Huntley Street, London WC1 6DD, UK

5 Centre for Safe Medication Practice and Research, Department of Pharmacology and Pharmacy, University of Hong Kong, Hong Kong, China 


\section{Background}

Neutropenia is a well-recognised complication of chemotherapy, associated with an increased risk of infection, febrile neutropenia (FN), and as a consequence, can lead to mortality [1]. Interventions to prevent neutropenic events (NEs) such as FN can reduce incidence and associated complications. Inventions include chemotherapy dose reductions and delays, prescribing prophylactic antibiotics and more commonly prescribing primary prophylaxis with colony-stimulating factors (CSFs). This latter strategy is favoured to maintain dose intensity. Guidelines are available that recommend the use of CSFs when a risk of FN is $20 \%$ or greater [2,3]. However, the start time and duration of treatment remain at the discretion of the patient's clinician.

In clinical practice, decisions on the best strategy to prevent NEs in patients treated with chemotherapy are challenging. With a diverse treatment population varying in weight, ethnicity, age, and co-morbidity, judgement has to be made on appropriate treatments and management strategies. Toxicity information from clinical trials is used to guide whether CSF prophylaxis is indicated [4]. However, data on neutropenic complications from clinical studies may not be representative of the wider population and this has caused uncertainty in using toxicity information as a guide [5].

Internationally recognised guidelines reflect this doubt, advising that individual patient factors such as age, and line of treatment should additionally be considered alongside toxicity information to guide management decisions $[2,6]$. Yet, inconsistencies exist in the reported studies for associations with factors such as increasing age and neutropenic events, where one study showed an increase in neutropenia risk and another reported a reduced risk [7, 8]. Additionally, there is no quantification of risk associated between factors and neutropenic events within guidelines, which is essential for clinical decision-making.

The aim of this review was to therefore investigate factors that have demonstrated influence on neutropenic episodes and synthesise their significance. There is already a recognition of the importance of the chemotherapy regimen and an additional understanding of other risk factors would enable clinicians to appropriately prescribe preventative measures.

\section{Methods}

\section{Search strategy and selection criteria}

This is a systematic review that includes a meta-analysis based on peer-reviewed academic articles. The Preferred Reporting Items for Systematic Reviews and Meta-Analysis (PRISMA) guidelines [9] were followed for reporting of the methods and findings. The review protocol was registered in the Prospero
International Prospective Register of Systematic Reviews (CRD42018097263).

Studies were identified through a literature search, guided by the Population-Intervention-Comparison-Outcomes (PICOs) framework, using MEDLINE, EMBASE and Cumulative Index to Nursing and Allied Health Literature (CINAHL) databases, from inception to December 1, 2017. An example of the search strategy is given in supplementary material. Reference lists of articles were reviewed to identify additional relevant publications.

Articles were screened against the inclusion criteria in two phases, by author PC, titles and abstracts followed by full texts; a duplicate screen of $10 \%$ of articles was screened by a second researcher (ML). Any conflict or uncertainty was resolved through consensus agreement with author YJ.

Studies were included if they were published in English and included human subjects aged 18 and over that were receiving cancer chemotherapy. We included studies that were systematic reviews, randomised controlled trials or observational studies. The studies must have quantitatively evaluated the association between individual factors and any NE, i.e. FN, FN admission, dose delays due to neutropenia or laboratory-tested myelosuppression. Exclusions included early phase pharmacological studies, where the purpose was to evaluate a drug or drug effect. Additionally, book reviews, opinion articles, editorial reviews and articles published in only abstract form were excluded.

A data extraction form was developed and piloted independently by two of the researchers (blinded) using a random sample of five articles. The following were extracted by the two researchers for each article: study design, method of data collection, setting, population characteristics (tumour group), method of analysis, all risk factors investigated, outcomes measured and strengths of association reported for significant factors. Data were extracted for the adjusted odds ratios (OR), relative risk (RR) and hazard ratios (HR), 95\% confidence intervals and $p$ values, where reported.

Methodological quality of studies was assessed for bias. As there were no randomised controlled studies that met the eligibility criteria, we used a published, modified version the Newcastle-Ottawa tool to assess the quality of studies [10] that provided a more comprehensive understanding for study quality and any bias that may exist [11], including handling of missing data. Quality was rated as high, moderate or low, if the total scores were greater than 17, 12-16 and less than 12 out of the total 21 , respectively. PC conducted all quality reviews with a second author (YJ), independently duplicating 5\% of the reviews with agreement on all double reviewed articles.

\section{Statistical analysis}

It is understood from previous studies that confounders can impact the strength of associations [12]. Hence, only studies 
which adjusted for confounders using appropriate statistical techniques were included in any meta-analysis. The pooled odds ratio was calculated for neutropenic events at different age groups and for one co-morbidity compared to zero, using random effects models. Due to the heterogeneity of the studies, it was not possible to aggregate other factors other than age and co-morbidity in the same way. The Q-test was performed to assess between-study heterogeneity, and calculated the $\mathrm{I}^{2}$ statistic, which expresses the percentage of the total observed variability due to study heterogeneity. A subgroup analysis was necessary to explore the variation of the effect of age on neutropenic events. In this subgroup, we only included articles that adjusted for the confounders, renal function and co-morbidity. All analyses were conducted in STATA v 15.1 SE.

\section{Results}

\section{Identification of articles}

The initial search returned 4415 published articles. Following title and abstract screening, 161 full-text articles were assessed against the inclusion and exclusion criteria, and 37 articles were included (Fig. 1). All identified articles were published between 2000 and 2017. The locations of these studies included the USA $(n=11)[7,8,13-21]$, Japan $(n=8)$ [22-29], the UK $(n=3)$ [30-32], Korea $(n=2)[33,34]$, France $(n=1)$ [35], Canada $(n=3)$ [36-38], Belgium $(n=1)$ [39], India $(n=1)$ [40], China $(n=1)$ [41], and Spain $(n=1)$ [42]. Other studies involved multiple countries either through collection utilising collaboration [43-45] or utilising data available from randomised controlled studies [46, 47].

FN was the primary outcome measure for most studies [8, 13-20, 22, 24-26, 28, 30-40, 42, 45-48]. Other outcome measures used were grade 3 or above neutropenia [7, 15, 16, $19,23,24,26,27,29,35,40-42,44,48]$, and dose delays and reductions [7, 24, 43].

Many studies included were in breast cancer, namely early breast cancer $[20,31,33,36-39,43,44]$ and a further 5 focussed on non-specific breast cancer [8, 13, 17, 21, 46]. Others investigated lung cancer patients [24, 27, 28, 30, 32, 41], patients with gynaecological malignancies [7, 14, 19], colorectal $[25,47]$, prostate $[22,26]$ and oesophageal cancers [29], myeloma [23], non-Hodgkin's lymphoma [18, 34, 35, 45], and glioblastoma [40]. Other studies grouped 3 or more tumour types together [13, 15, 16, 42].

Overall, the factors identified were concordant with those found in a similar systematic review [49] and could be grouped into patient-, cancer- or treatment-related factors. Supplementary Table 2 outlines studies not included in the meta-analysis. Some authors aimed to develop predictive risk models, using findings from their research $[16,17,25,39,45,48]$. These models would enable clinicians to score patient-related factors and calculate individual patient risk. Other authors focussed in detail into specific factors such as co-morbidity [15] or genetic influences [25] and articles only reported details of these associated hazard or odds ratios, despite recognising confounding factors were associated with the event. The 12 articles in Table 1 were included in our meta-analysis and the table describes details of confounders used in analysis.

Quality assessment identified that a number of studies included overall did not document their use of missing data [7, $8,15,19,23,27-29,34,37,41,42,46,50]$ and some failed to meet the minimum required sample size necessary to draw conclusions [23-27, 29]. In addition, when using univariable methods to choose factors to build into the multivariable models, some studies used a standard $95 \%$ significance level test $[7,8,19,23,24,26,28,29,34,37,42,50]$. Although this is a recognised standard in many circumstances, in these types of studies, it is preferable to use a less rigorous cut-off to include factors that may become significant when adjusted for confounders. Despite this, for the majority, quality was high in all other domains of the assessment.

Of the patient-related factors, age was studied by 17 authors $[7,8,16,18,19,25,26,28,29,33,34,37,41,43,44,46$, 51] with the majority finding older age to significantly increase NEs [8, 16, 18, 19, 25, 26, 41, 43-46, 50]. Conversely, one study involving 635 patients with gynaecological malignancies concluded that lower age increased the risk of NEs [7]. Additionally, in two other studies, age was found to be non-significant in univariable analysis $[17,39]$ and therefore was not investigated through multivariable methods. Pettengell et al. [45] found age to be significant in their risk model development study but actually noted that it could be interchanged with a marker of renal function. There was variation in the way that age was analysed: authors used either linear chronological age [25], or dichotomised age, such as using a threshold of greater than 65 years $[8,16,18]$.

A meta-analysis of age where ORs were available and pooled yielded a combined OR of 1.2 (1.06-1.36) (Fig. 2). This included studies from a number of tumour groups, and in some cases, there was no adjustment for the confounders' renal function and co-morbidity which may limit clinical acceptance. Additionally, when $\mathrm{I}^{2}$ was calculated, a high degree of heterogeneity was identified. A subanalysis that only included results from studies that adjusted for important confounders such as co-morbidity and either renal or liver function [16, 33, 44] (Fig. 3) yielded an OR of 1.39 (1.11, 1.76) (Fig. 4), with an acceptable level of heterogeneity $\left(\mathrm{I}^{2}=24.1 \%\right)$. These 3 studies selected in the analysis included data from 4814 patients of which 2497 patients were treated for breast cancer.

A number of studies investigating co-morbidity $[7,8$, 16-18, 26, 29, 33, 34, 41, 44, 45] found it to be a significant factor. However, we were only able to pool results from two articles due to the diverse methods in which co-morbidity was 
Fig. 1 PRISMA flow diagram

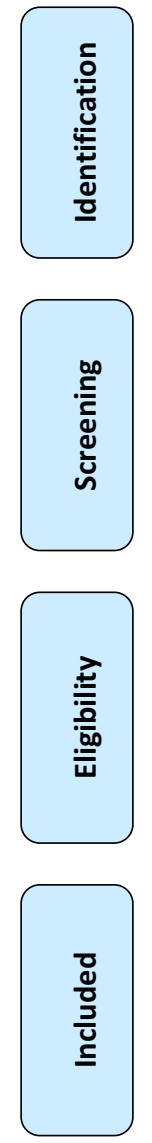

recorded. Within these, four independent studies were reported. Agiro et al. reported OR for patients receiving 3 separate chemotherapy regimens, where events and controls were independent for the different treatment groups. The aggregation of the four studies yielded an overall OR of 1.54 (CI 1.092.09) (see Fig. 4). This analysis included 9189 curative breast cancer patients. All studies in this meta-analysis included neutropenia-related hospitalisations encompassing $\mathrm{FN}$ in addition to treatment delays as measures of outcome. To add to this combined result, one very large study including 19,160 patients detailed the effect of individual co-morbidities and found that having three or more other conditions produced a HR of 1.73 (1.33-2.26) for FN. Conversely, a non-significant result was reported for grade 4 neutropenias [15], highlighting differences in mechanisms between NS and NEs.

Findings from smaller studies investigating markers of myelosuppression such as neutrophil, platelet and white cell counts or levels of haemoglobin prior to chemotherapy initiation [24, 25, 27, 29] were limited by inadequate sample sizes. Two larger studies $[16,44]$ did find that reduced white cell counts prior to treatment would increase the risk of NS; however, threshold values were unavailable from the articles. One study involving 509 breast cancer patients did conclude that a neutrophil value of $<2 \times 10^{9} / \mathrm{L}$ at baseline would result in a 4.2-fold increase in the risk of any neutropenic complication [46]. Similarly, in a study investigating cycle $1 \mathrm{FN}$ in 577 patients with non-Hodgkin's lymphoma [18], a pre-treatment haemoglobin level of $<12 \mathrm{~g} / \mathrm{L}$ resulted in a hazard ratio of 1.44 (1.03-2). Results could not be pooled due to differences in measurements and dichotomisation.

\section{Discussion}

The aim of this review was to evaluate, via a meta-analysis, the associations between factors that have demonstrated influence on NEs, in order to guide future management of chemotherapy patients. A number of factors were frequently investigated and reported to be associated with NEs, including age, co-morbidity and baseline bone marrow suppression. We were 


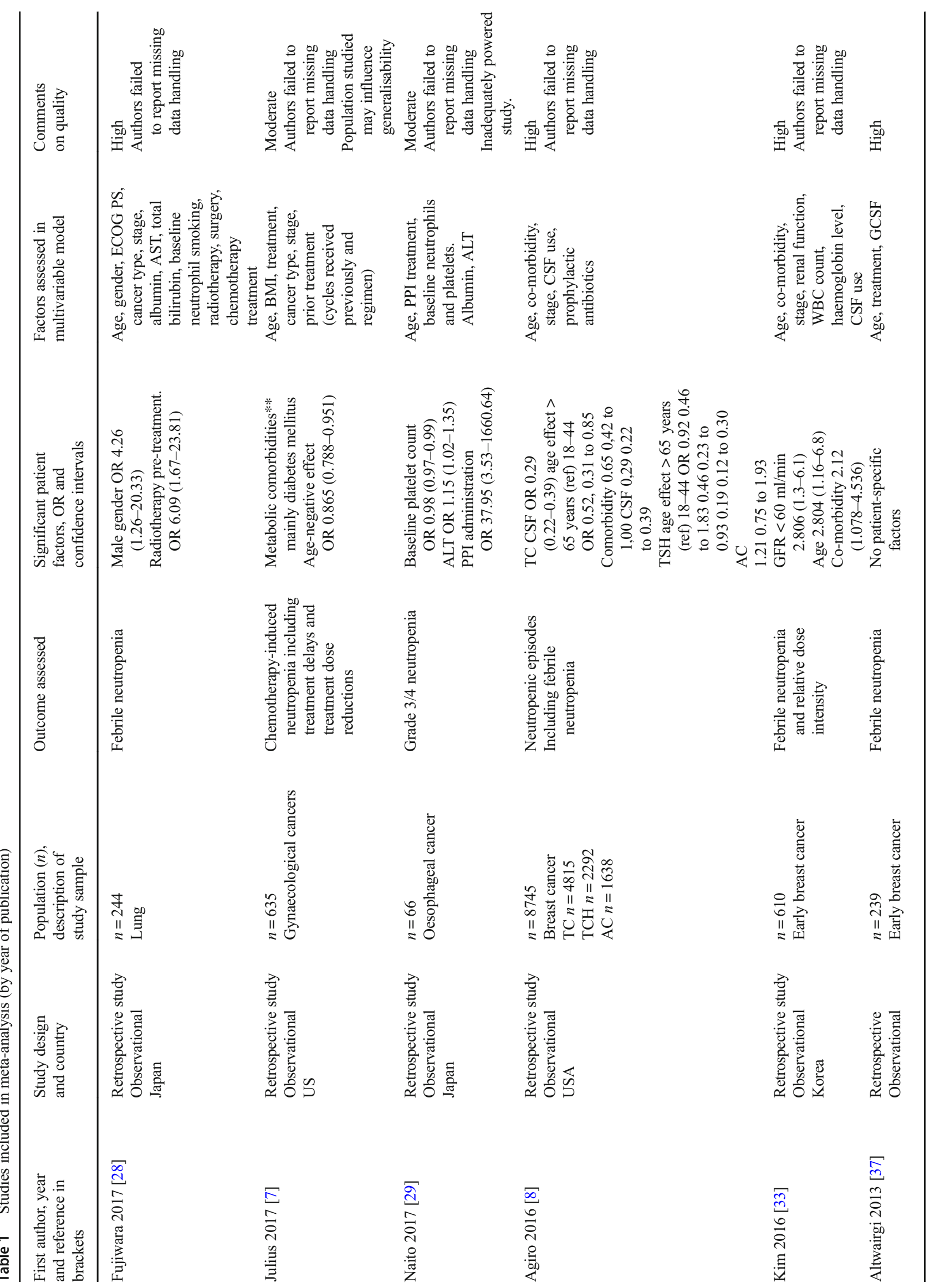




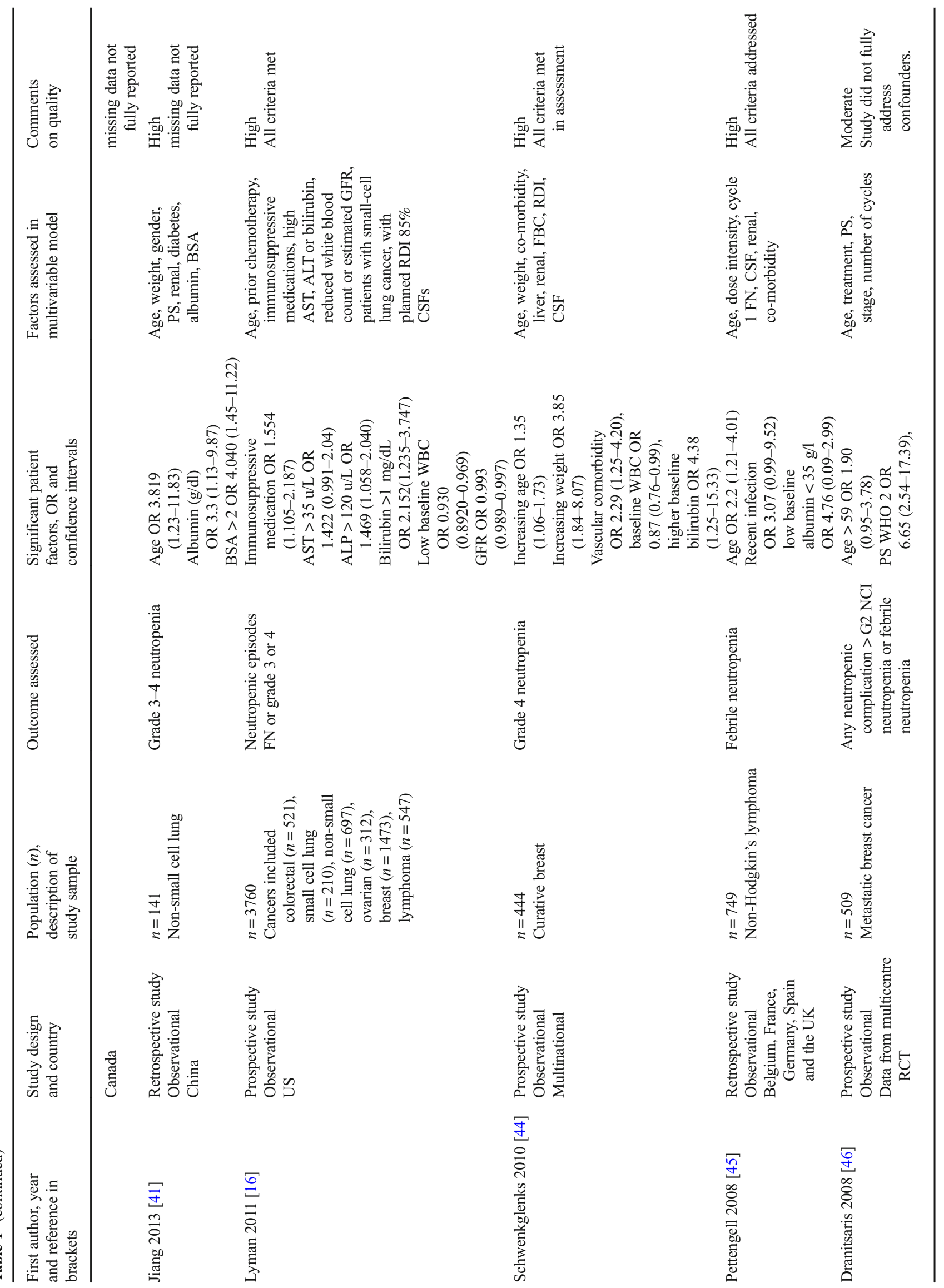




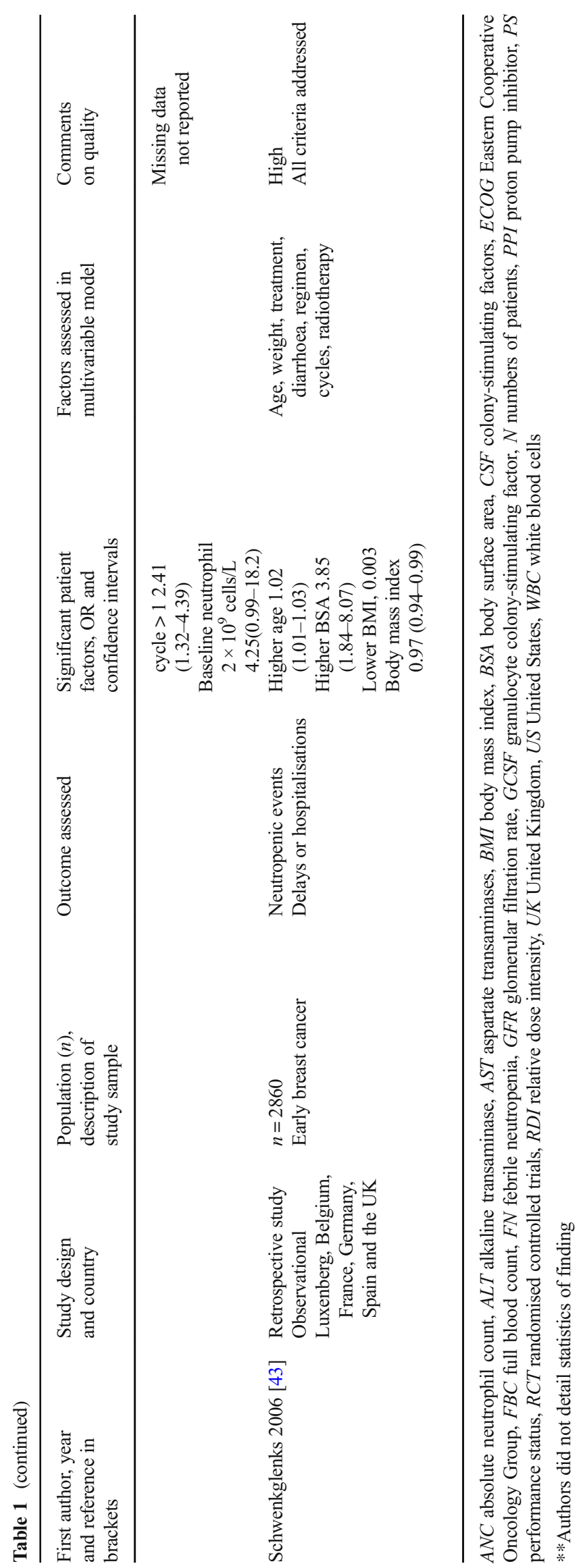




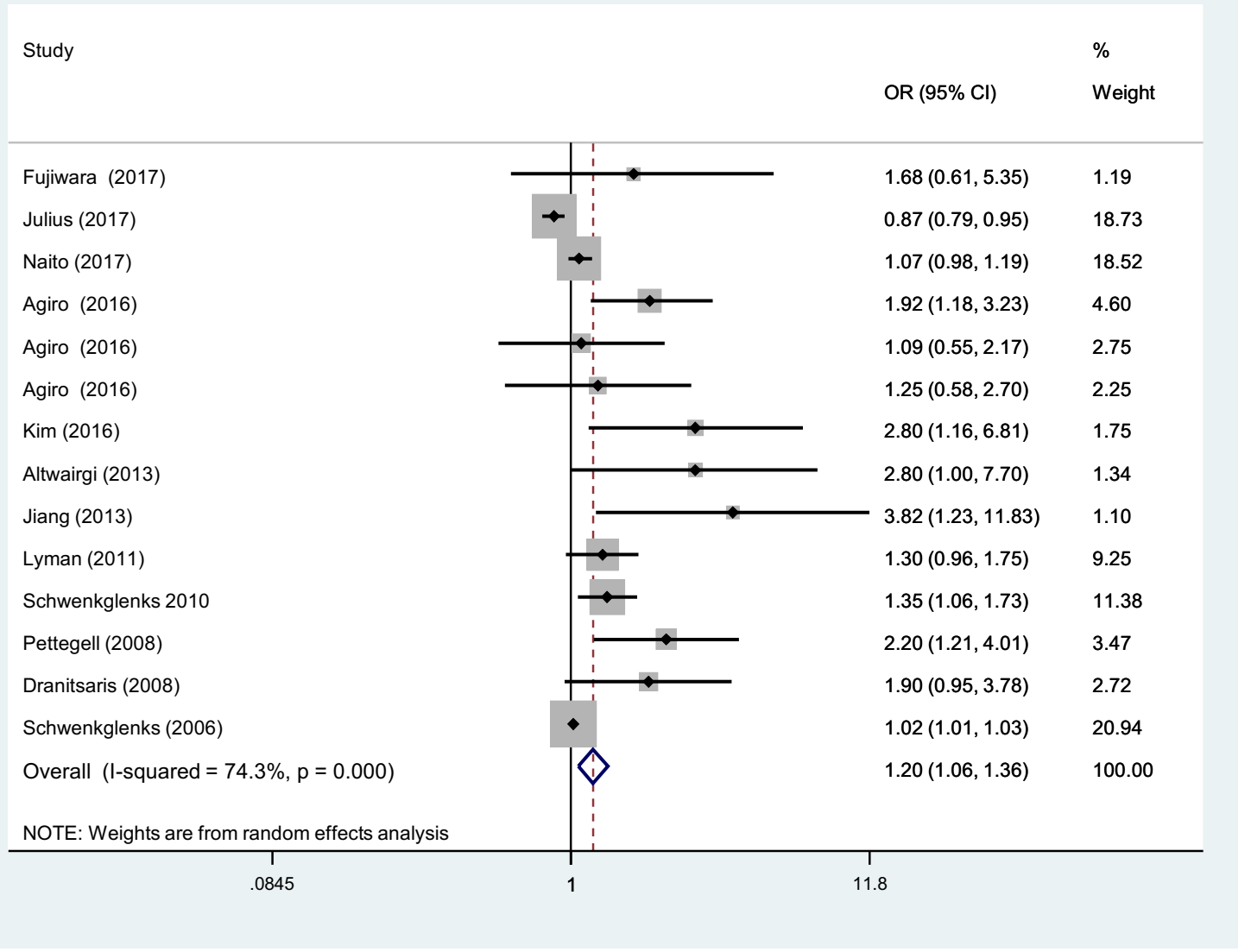

Fig. 2 Meta-analysis showing pooled odds of neutropenic events with ages $>65$ years. OR, odds ratios; CI, confidence intervals

able to aggregate the reported OR from included studies for both age and co-morbidity and determine the pooled effect. We found that increased age and the presence of just one comorbidity increase the occurrence of NEs by approximately 40 and $50 \%$ respectively; these findings should be used to guide the management of patients.

Similar factors encountered in our study were also described in another systematic review conducted in 2014 [49]. However, in our review, by adding quantifications to factors related to NEs, we have enhanced understanding of the importance of personalised care. This is particularly relevant as we approach an era of pre-determined electronic prescribing protocols for chemotherapy and supportive care. The implications of our findings are more prominent in treatments that fall marginally short of the $20 \%$ neutropenic risk threshold that currently indicates use of CSFs. A combined OR of 1.39 for an age above 65 and 1.54 for one co-morbidity (compared with none) was found. In practice, the results could determine individualised CSF prescribing rather than simply using published toxicity data from clinical trials. In the cases of early breast cancer, where treatments such as docetaxel/ cyclophosphamide in clinical trials have demonstrated a moderate risk of NEs of 15\% [52], clinicians may want to consider primary prophylaxis.
Our findings cannot yet be fully incorporated into practice guidelines, primarily because there is yet to be a strong, prospective study that evaluates all factors that may affect NEs. These factors include those such as performance status, severity of co-morbidity and ethnicity. The recently updated NCCN guidance has acknowledged the increased risk with advanced age [53]; however, age could simply be a proxy measure for frailty [54] or organ function. Within the meta-analysis, only studies adjusting for confounders were included but frailty was not studied by any author, which limits our findings. The diversity in study methods and criteria for inclusion limited us to only being able to pool data for one co-morbid condition compared to zero. Equally, we could not aggregate results of baseline bone marrow function or the effect of renal and liver function tests. The influence of gender has also been recently highlighted as an area where further research is required [55] and should also be considered when assigning treatment. The majority of studies that were appropriate for meta-analysis included women receiving treatment for breast cancer. This limited evaluation of gender and may also limit the findings of the study to patients treated with breast cancer.

Despite only including studies that included similar confounders, we found heterogeneity was inherently present due to differences in collection methods. One source of 


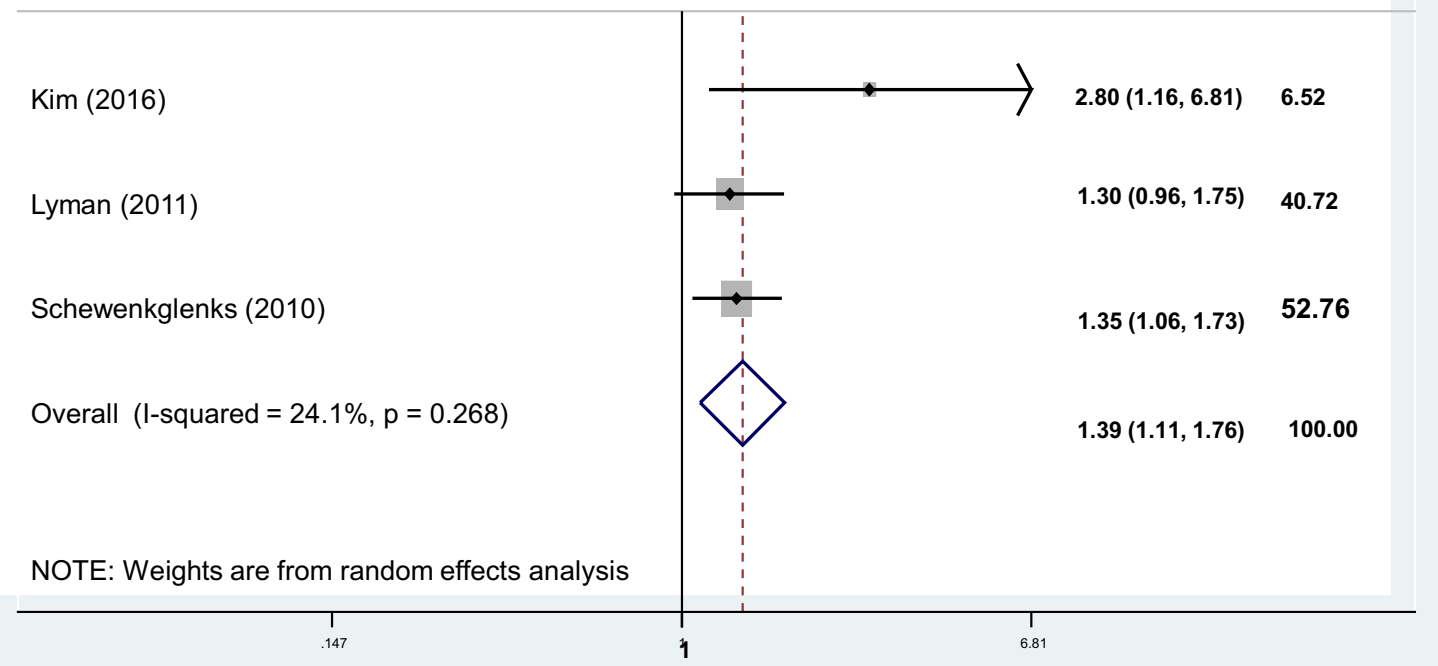

Fig. 3 Subgroup analysis showing pooled odds of neutropenic events with $>65$ years. Only studies adjusting for confounders included. OR, odds ratios; CI, confidence intervals

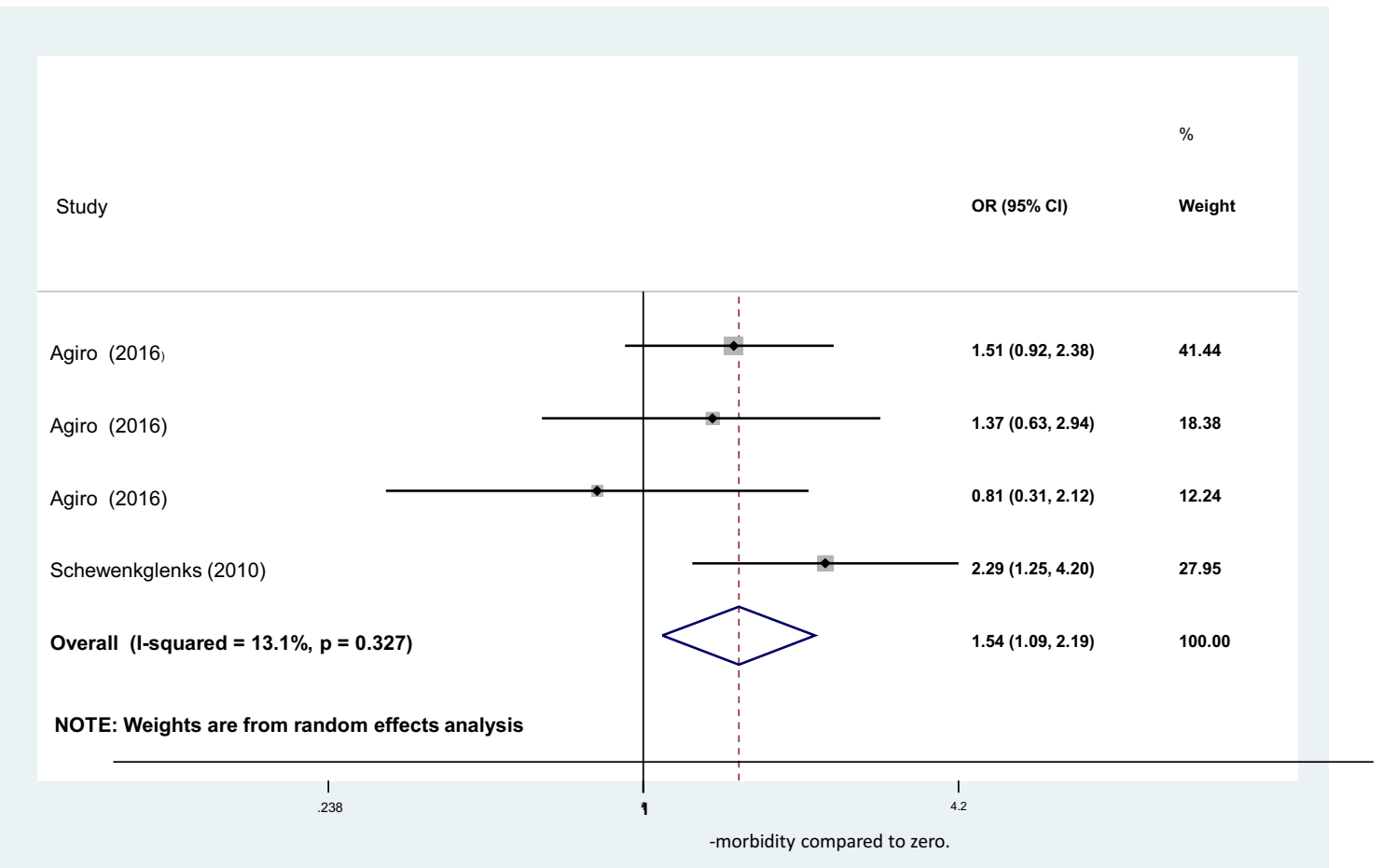

Fig. 4 Meta-analysis showing pooled odds of neutropenic events with one co-morbidity compared to zero. OR, odds ratios; CI, confidence intervals 
heterogeneity was our grouping of grade $3-4 \mathrm{NE}$ and $\mathrm{FN}$ as outcome measures. Interestingly, there were no reports of mortality in any of the articles, which may be an effect of the collection methods. Further work is needed to define how neutropenic episodes translate to mortality following the developments in rescue medications such as CSFs.

A prospective study incorporating the findings of our work could guide the development of a risk prediction model. We found a number of model development studies; however, many were excluded from our review as individual OR and HR were not reported. We have identified weaknesses with some of the risk prediction models included in our analysis, consistent with other models developed for use in cancer patients, limiting their current use.

Neutropenia is one of the most common and most dangerous AEs of chemotherapy. For this reason, a strategy to prevent the event occurring is essential. Trial data of new treatment regimens can help us to understand the effects of treatment on the bone marrow. However, these studies are often undertaken in a controlled group of patients and it is difficult to assess other patient-related factors that increase the risk.

\section{Conclusions}

Our study has demonstrated that there are many patientrelated factors that have influence on NEs. By determining the magnitude of risk of advanced age and co-morbidity, we have enhanced current guidance. However, further work is urgently needed in developing a comprehensive risk model to guide better patient management.

Acknowledgements We would like to thank Ms. Misha Ladva (ML) for her contributions to the article screening and extraction.

Funding information The work was supported by a National Institute for Health Research award; author PC is a Doctoral Research Fellow funded by the NIHR Academy (Project reference DRF 2017-10-016).

\section{Compliance with ethical standards}

Conflict of interest The authors declare that they have no conflict of interest.

Disclaimer The views expressed are those of the authors and are not necessarily those of the NHS, the NIHR or the Department of Health and Social Care. The authors have full control over data extracted and agree for this to be reviewed on request.

Open Access This article is distributed under the terms of the Creative Commons Attribution 4.0 International License (http:// creativecommons.org/licenses/by/4.0/), which permits unrestricted use, distribution, and reproduction in any medium, provided you give appropriate credit to the original author(s) and the source, provide a link to the Creative Commons license, and indicate if changes were made.

\section{References}

1. Lyman GH, Kuderer NM, Djulbegovic B (2002) Prophylactic granulocyte colony-stimulating factor in patients receiving doseintensive cancer chemotherapy: a meta-analysis. Am J Med 112: 406-411

2. Smith TJ, Bohlke K, Lyman GH, Carson KR, Crawford J, Cross SJ, Goldberg JM, Khatcheressian JL, Leighl NB, Perkins CL, Somlo G, Wade JL, Wozniak AJ, Armitage JO, American Society of Clinical (2015) Recommendations for the use of WBC growth factors: American Society of Clinical Oncology clinical practice guideline update. J Clin Oncol 33:3199-3212

3. Klastersky J, de Naurois J, Rolston K, Rapoport B, Maschmeyer G, Aapro M, Herrstedt J, Committee EG (2016) Management of febrile neutropaenia: ESMO clinical practice guidelines. Ann Oncol 27:v111-v118

4. Smith TJ, Hillner BE (2016) Real-world conundrums and biases in the use of white cell growth factors. Am Soc Clin Oncol Educ Book 35:e524-e527

5. Khoja L, Atenafu EG, Ye Q, Gedye C, Chappell M, Hogg D, Butler MO, Joshua AM (2016) Real-world efficacy, toxicity and clinical management of ipilimumab treatment in metastatic melanoma. Oncol Lett 11:1581-1585

6. McNeil C (2005) NCCN guidelines advocate wider use of colonystimulating factor. J Natl Cancer Inst 97:710-711

7. Julius JM, Hammerstrom A, Wei C, Rajesh R, Bodurka DC, Kurian S, Smith JA (2017) Defining the impact of the use of granulocyte colony stimulating factors on the incidence of chemotherapyinduced neutropenia in patients with gynecologic malignancies. J Oncol Pharm Pract 23:121-127

8. Agiro A, Ma Q, Acheson AK, Wu SJ, Patt DA, Barron JJ, Malin JL, Rosenberg A, Schilsky RL, Lyman GH (2016) Risk of neutropeniarelated hospitalization in patients who received colony-stimulating factors with chemotherapy for breast cancer. J Clin Oncol 19:19

9. Liberati A, Altman DG, Tetzlaff J, Mulrow C, Gotzsche PC, Ioannidis JP, Clarke M, Devereaux PJ, Kleijnen J, Moher D (2009) The PRISMA statement for reporting systematic reviews and meta-analyses of studies that evaluate healthcare interventions: explanation and elaboration. BMJ 339:b2700

10. Luchini C, Stubbs B, Solmi M, Veronese N (2017) Assessing the quality of studies in meta-analyses: advantages and limitations of the Newcastle Ottawa Scale. World J Metaanal 5:80

11. Quigley JM, Thompson JC, Halfpenny NJ, Scott DA (2019) Critical appraisal of nonrandomized studies-a review of recommended and commonly used tools. J Eval Clin Pract 25:44-52

12. Li L, Kleinman K, Gillman MW (2014) A comparison of confounding adjustment methods with an application to early life determinants of childhood obesity. J Dev Orig Health Dis 5:435-447

13. Li X, Luthra R, Morrow PK, Fisher MD, Reiner M, Barron RL, Langeberg WJ (2016) Comorbidities among patients with cancer who do and do not develop febrile neutropenia during the first chemotherapy cycle. J Oncol Pharm Pract 22:679-689

14. Phippen NT, Lowery WJ, Barnett JC, Hall LA, Landt C, Leath CA 3rd (2011) Evaluation of the Patient-Generated Subjective Global Assessment (PG-SGA) as a predictor of febrile neutropenia in gynecologic cancer patients receiving combination chemotherapy: a pilot study. Gynecol Oncol 123:360-364

15. Chao C, Page JH, Yang SJ, Rodriguez R, Huynh J, Chia VM (2014) History of chronic comorbidity and risk of chemotherapy-induced febrile neutropenia in cancer patients not receiving G-CSF prophylaxis. Ann Oncol 25:1821-1829

16. Lyman GH, Kuderer NM, Crawford J, Wolff DA, Culakova E, Poniewierski MS, Dale DC (2011) Predicting individual risk of neutropenic complications in patients receiving cancer chemotherapy. Cancer 117:1917-1927 
17. Hosmer W, Malin J, Wong M (2011) Development and validation of a prediction model for the risk of developing febrile neutropenia in the first cycle of chemotherapy among elderly patients with breast, lung, colorectal, and prostate cancer. Support Care Cancer 19:333-341

18. Lyman GH, Morrison VA, Dale DC, Crawford J, Delgado DJ, Fridman M, O.W. Group, A.N.C.S. Group (2003) Risk of febrile neutropenia among patients with intermediate-grade nonHodgkin's lymphoma receiving CHOP chemotherapy. Leuk Lymphoma 44:2069-2076

19. Laskey RA, Poniewierski MS, Lopez MA, Hanna RK, Secord AA, Gehrig PA, Lyman GH, Havrilesky LJ (2012) Predictors of severe and febrile neutropenia during primary chemotherapy for ovarian cancer. Gynecol Oncol 125:625-630

20. Hurria A, Brogan K, Panageas KS, Pearce C, Norton L, Jakubowski A, Zauderer M, Howard J, Hudis C (2005) Patterns of toxicity in older patients with breast cancer receiving adjuvant chemotherapy. Breast Cancer Res Treat 92:151-156

21. Rivera E, Erder MH, Moore TD, Shiftan TL, Knight CA, Fridman M, Brannan C, Danel-Moore L, Hortobagyi GN, G. Risk Model Study (2003) Targeted filgrastim support in patients with earlystage breast carcinoma: toward the implementation of a risk model. Cancer 98:222-228

22. Shiota M, Yokomizo A, Takeuchi A, Kiyoshima K, Inokuchi J, Tatsugami K, Naito S (2014) Risk factors for febrile neutropenia in patients receiving docetaxel chemotherapy for castrationresistant prostate cancer. Support Care Cancer 22:3219-3226

23. Mitani Y, Usami E, Kimura M, Nakao T, Okada K, Matsuoka T, Kokuryou T, Yoshimura T, Yamakawa M (2016) Risk factors for neutropenia with lenalidomide plus dexamethasone therapy for multiple myeloma. Pharmazie 71:349-351

24. Ikesue H, Watanabe H, Hirano M, Chikamori A, Suetsugu K, Ryokai Y, Egashira N, Yamada T, Ikeda M, Iwama E, Harada T, Takayama K, Nakanishi Y, Masuda S (2015) Risk factors for predicting severe neutropenia induced by pemetrexed plus carboplatin therapy in patients with advanced non-small cell lung cancer. Biol Pharm Bull 38:1192-1198

25. Ichikawa W, Uehara K, Minamimura K, Tanaka C, Takii Y, Miyauchi H, Sadahiro S, Fujita K, Moriwaki T, Nakamura M, Takahashi T, Tsuji A, Shinozaki K, Morita S, Ando Y, Okutani Y, Sugihara M, Sugiyama T, Ohashi Y, Sakata Y (2015) An internally and externally validated nomogram for predicting the risk of irinotecan-induced severe neutropenia in advanced colorectal cancer patients. Br J Cancer 112:1709-1716

26. Shigeta K, Kosaka T, Yazawa S, Yasumizu Y, Mizuno R, Nagata H, Shinoda K, Morita S, Miyajima A, Kikuchi E, Nakagawa K, Hasegawa S, Oya M (2015) Predictive factors for severe and febrile neutropenia during docetaxel chemotherapy for castration-resistant prostate cancer. Int J Clin Oncol 20:605-612

27. Watanabe H, Ikesue H, Oshiro M, Nagata K, Mishima K, Takada A, Suetsugu K, Sueyasu M, Egashira N, Harada T, Takayama K, Nakanishi Y, Oishi R (2012) Risk factors for predicting severe neutropenia induced by amrubicin in patients with advanced lung cancer. Chemotherapy 58:419-425

28. Fujiwara T, Kenmotsu H, Naito T, Kawamura T, Mamesaya N, Kotake M, Kobayashi H, Omori S, Nakashima K, Wakuda K, Ono A, Taira T, Murakami H, Omae K, Mori K, Endo M, Takahashi T (2017) The incidence and risk factors of febrile neutropenia in chemotherapy-naive lung cancer patients receiving etoposide plus platinum. Cancer Chemother Pharmacol 79:12291237

29. Naito M, Yamamoto T, Shimamoto C, Miwa Y (2017) Retrospective analysis of the risk factors for grade IV neutropenia in oesophageal cancer patients treated with a docetaxel, cisplatin, and 5-fluorouracil regimen. Chemotherapy 62:215-224
30. Jenkins P, Scaife J, Freeman S (2012) Validation of a predictive model that identifies patients at high risk of developing febrile neutropaenia following chemotherapy for breast cancer. Ann Oncol 23:1766-1771

31. Jenkins P, Freeman S (2009) Pretreatment haematological laboratory values predict for excessive myelosuppression in patients receiving adjuvant FEC chemotherapy for breast cancer. Ann Oncol 20: $34-40$

32. Crawford J, Glaspy JA, Stoller RG, Tomita DK, Vincent ME, McGuire BW, Ozer H (2005) Final results of a placebo-controlled study of filgrastim in small-cell lung cancer: exploration of risk factors for febrile neutropenia. Support Cancer Ther 3:36-46

33. Kim HS, Lee SY, Kim JW, Choi YJ, Park IH, Lee KS, Seo JH, Shin SW, Kim YH, Kim JS, Park KH (2016) Incidence and predictors of febrile neutropenia among early-stage breast cancer patients receiving anthracycline-based chemotherapy in Korea. Oncology 91: 274-282

34. Choi YW, Jeong SH, Ahn MS, Lee HW, Kang SY, Choi JH, Jin UR, Park JS (2014) Patterns of neutropenia and risk factors for febrile neutropenia of diffuse large B-cell lymphoma patients treated with rituximab-CHOP. J Korean Med Sci 29:1493-1500

35. Voog E, Bienvenu J, Warzocha K, Moullet I, Dumontet C, Thieblemont C, Monneret G, Gutowski MC, Coiffier B, Salles G (2000) Factors that predict chemotherapy-induced myelosuppression in lymphoma patients: role of the tumor necrosis factor ligand-receptor system. J Clin Oncol 18:325-331

36. Assi H, Murray J, Boyle L, Rayson D (2014) Incidence of febrile neutropenia in early stage breast cancer patients receiving adjuvant FEC-D treatment. Support Care Cancer 22:3227-3234

37. Altwairgi AK, Hopman WM, Mates M (2013) Real-world impact of granulocyte-colony stimulating factor on febrile neutropenia. Curr Oncol 20:e171-e179

38. Shirdel EA, Korenberg MJ, Madarnas Y (2011) Neutropenia prediction based on first-cycle blood counts using a FOS-3NN classifier. Adv Bioinforma 2011:172615

39. Pfeil AM, Vulsteke C, Paridaens R, Dieudonne AS, Pettengell R, Hatse S, Neven P, Lambrechts D, Szucs TD, Schwenkglenks M, Wildiers H (2014) Multivariable regression analysis of febrile neutropenia occurrence in early breast cancer patients receiving chemotherapy assessing patient-related, chemotherapy-related and genetic risk factors. BMC Cancer 14:201

40. Gupta T, Mohanty S, Moiyadi A, Jalali R (2013) Factors predicting temozolomide induced clinically significant acute hematologic toxicity in patients with high-grade gliomas: a clinical audit. Clin Neurol Neurosurg 115:1814-1819

41. Jiang N, Chen XC, Zhao Y (2013) Analysis of the risk factors for myelosuppression after concurrent chemoradiotherapy for patients with advanced non-small cell lung cancer. Support Care Cancer 21: 785-791

42. Lopez-Pousa A, Rifa J, Casas de Tejerina A, Gonzalez-Larriba JL, Iglesias C, Gasquet JA, Carrato A, D.S. Group (2010) Risk assessment model for first-cycle chemotherapy-induced neutropenia in patients with solid tumours. Eur J Cancer Care (Engl) 19:648-655

43. Schwenkglenks M, Jackisch C, Constenla M, Kerger JN, Paridaens R, Auerbach L, Bosly A, Pettengell R, Szucs TD, Leonard R (2006) Neutropenic event risk and impaired chemotherapy delivery in six European audits of breast cancer treatment. Support Care Cancer 14:901-909

44. Schwenkglenks M, Pettengell R, Jackisch C, Paridaens R, Constenla M, Bosly A, Szucs TD, Leonard R (2011) Risk factors for chemotherapy-induced neutropenia occurrence in breast cancer patients: data from the INC-EU Prospective Observational European Neutropenia Study. Support Care Cancer 19:483-490

45. Pettengell R, Bosly A, Szucs TD, Jackisch C, Leonard R, Paridaens R, Constenla M, Schwenkglenks M, G. Impact of Neutropenia in Chemotherapy-European Study (2009) Multivariate analysis of 
febrile neutropenia occurrence in patients with non-Hodgkin lymphoma: data from the INC-EU Prospective Observational European Neutropenia Study. Br J Haematol 144:677-685

46. Dranitsaris G, Rayson D, Vincent M, Chang J, Gelmon K, Sandor D, Reardon G (2008) Identifying patients at high risk for neutropenic complications during chemotherapy for metastatic breast cancer with doxorubicin or pegylated liposomal doxorubicin: the development of a prediction model. Am J Clin Oncol 31:369-374

47. Meyerhardt JA, Kwok A, Ratain MJ, McGovren JP, Fuchs CS (2004) Relationship of baseline serum bilirubin to efficacy and toxicity of single-agent irinotecan in patients with metastatic colorectal cancer. J Clin Oncol 22:1439-1446

48. Rivera E, Erder MH, Fridman M, Frye D, Hortobagyi GN (2003) First-cycle absolute neutrophil count can be used to improve chemotherapy-dose delivery and reduce the risk of febrile neutropenia in patients receiving adjuvant therapy: a validation study. Breast Cancer Res 5:R114-R120

49. Lyman GH, Abella E, Pettengell R (2014) Risk factors for febrile neutropenia among patients with cancer receiving chemotherapy: a systematic review. Crit Rev Oncol Hematol 90:190-199

50. Kim CG, Sohn J, Chon H, Kim JH, Heo SJ, Cho H, Kim IJ, Kim SI, Park S, Park HS, Kim GM (2016) Incidence of febrile neutropenia in Korean female breast cancer patients receiving preoperative or postoperative doxorubicin/cyclophosphamide followed by docetaxel chemotherapy. J Breast Cancer 19:76-82

51. Pettengell R, Schwenkglenks M, Leonard R, Bosly A, Paridaens R, Constenla M, Szucs TD, Jackisch C, G. Impact of Neutropenia in
Chemotherapy-European Study (2008) Neutropenia occurrence and predictors of reduced chemotherapy delivery: results from the INC-EU prospective observational European neutropenia study. Support Care Cancer 16:1299-1309

52. Jones S, Holmes FA, O'Shaughnessy J, Blum JL, Vukelja SJ, McIntyre KJ, Pippen JE, Bordelon JH, Kirby RL, Sandbach J, Hyman WJ, Richards DA, Mennel RG, Boehm KA, Meyer WG, Asmar L, Mackey D, Riedel S, Muss H, Savin MA (2009) Docetaxel with cyclophosphamide is associated with an overall survival benefit compared with doxorubicin and cyclophosphamide: 7-year follow-up of US oncology research trial 9735. J Clin Oncol 27:1177-1183

53. National Cancer Comprehensive Network (2019) Prevention and treatment of cancer-related infections https://www.nccn.org/ professionals/physician_gls/\#supportive. Accessed 01 Nov 2018

54. Belani CP, Fossella F (2005) Elderly subgroup analysis of a randomized phase III study of docetaxel plus platinum combinations versus vinorelbine plus cisplatin for first-line treatment of advanced nonsmall cell lung carcinoma (TAX 326). Cancer 104:2766-2774

55. Ozdemir BC, Csajka C, Dotto GP, Wagner AD (2018) Sex differences in efficacy and toxicity of systemic treatments: an undervalued issue in the era of precision oncology. J Clin Oncol $36: 2680-2683$

Publisher's note Springer Nature remains neutral with regard to jurisdictional claims in published maps and institutional affiliations. 